\title{
Investigation of ULF magnetic anomaly during Izu earthquake swarm and Miyakejima volcano eruption at summer 2000, Japan
}

\author{
A. Kotsarenko ${ }^{1}$, O. Molchanov ${ }^{2}$, M. Hayakawa ${ }^{3}$, S. Koshevaya ${ }^{4}$, V. Grimalsky ${ }^{5}$, R. Pérez Enríquez ${ }^{1}$, and J. A. López \\ Cruz-Abeyro ${ }^{1}$ \\ ${ }^{1}$ Centro de Geociencias, Juriquilla, UNAM, Apdo Postal 1-742, Centro Queretaro, Querétaro, C.P. 76001, Mexico \\ ${ }^{2}$ Institute of the Physics of the Earth, Moscow, Russia \\ ${ }^{3}$ University of Electro Communications, Chofu 1-5-1, Tokyo 182-8585, Japan \\ ${ }^{4}$ CIICAp, UAEM, Cuernavaca, Morelos, Mexico \\ ${ }^{5}$ INAOE, Tonantzintla, P.O.51\&216, C.P. 72000, Puebla, Pue., Mexico
}

Received: 30 June 2004 - Revised: 7 December 2004 - Accepted: 13 December 2004 - Published: 5 January 2005

Part of Special Issue "Precursory phenomena, seismic hazard evaluation and seismo-tectonic electromagnetic effects"

\begin{abstract}
Results of the ULF electromagnetic emission during the Izu, 2000 earthquake (EQ) swarm and Miyake volcano eruptions during the summer period of 2000 are presented and analyzed. The analysis of the obtained data has been performed in 3 main directions: traditional statistical analysis, i.e. analysis of time dynamics of spectral density, polarization ratios and their derivatives, Principal Component Analysis, and Fractal Analysis. The statistical characteristics were studied at different frequency sub-bands in a frequency range $10^{-3}-0.3 \mathrm{~Hz}$. These methods of data processing are described and the obtained results are illustrated and discussed. Some peculiarities of the obtained results such as the rise of the second principal component and the rise of the fractal index can be interpreted as possible shorttime precursors.
\end{abstract}

\section{Introduction}

Recent studies of EQs and volcanic eruptions emphasize the electromagnetic phenomena to be a very promising candidate for their forecasting. The application of electromagnetic methods in addition to the traditional methods of seismology becomes important since some strong EQs (Loma Prieta, Kobe and Guam) occurred without any pronounced foreshock.

Different long-term observatories are already in operation in Japan (Hayakawa, et al., 1996; Uyeda et al., 2000), Russia (Ismaguilov et al., 2001), Greece (Varotsos et al., 1996), U.S. (Fraser-Smith, et al., 1990) etc. and the EM phenom-

Correspondence to: A. Kotsarenko

(kotsarenko@geociencias.unam.mx) ena is a subject of observation by means of different instrumental infrastructure and study with a robust mathematical technique.

We focused our interest on the ULF part of the EM spectrum $\left(f=10^{-6}-10 \mathrm{~Hz}\right)$. Numerous advanced studies including the recent ones (Ismaguilov et al., 2001; Hattori et al., 2002; Smirnova et al., 2004) prove the reliability of some applied algorithms and data processing techniques. In addition to that, the ULF part of electromagnetic emission, generated by the earthquake, can be recorded at the Earth's surface by modern magnetometers without significant attenuation if they are generated at typical earthquake nucleation depths $(\sim 10 \mathrm{~km})$. Higher frequencies would have smaller skin depths and, therefore, greater attenuation before reaching the Earth's surface. Finally, the ULF geomagnetic equipment does not require complicated maintenance. The main aim of our study is to find a statistical correlation between seismic and EM activities before and during the summer 2000 Izu EQ swarm.

\section{Description of the dynamics of Izu EQ swarm and Oyama volcano eruption}

The first alarm of the approaching eruption of Oyama volcano, Miyakejima Island $\left(34.09^{\circ} \mathrm{N}, 139.51^{\circ} \mathrm{E}\right.$, approximately at $150 \mathrm{~km}$ southwest from Tokyo) came from the Japan Meteorological Agency on 26 June at 18:30 LT. The warnings were based on the shallow $(\sim 10-15 \mathrm{~km})$ dense EQ swarm under the island, migrating northwest (Fig. 1a from Earthquake Research institute, University of Tokyo ERI, Toda et al., 2002). In July, new swarms were detected at the northern and southern parts of island (Figs. 1b and 1c), 

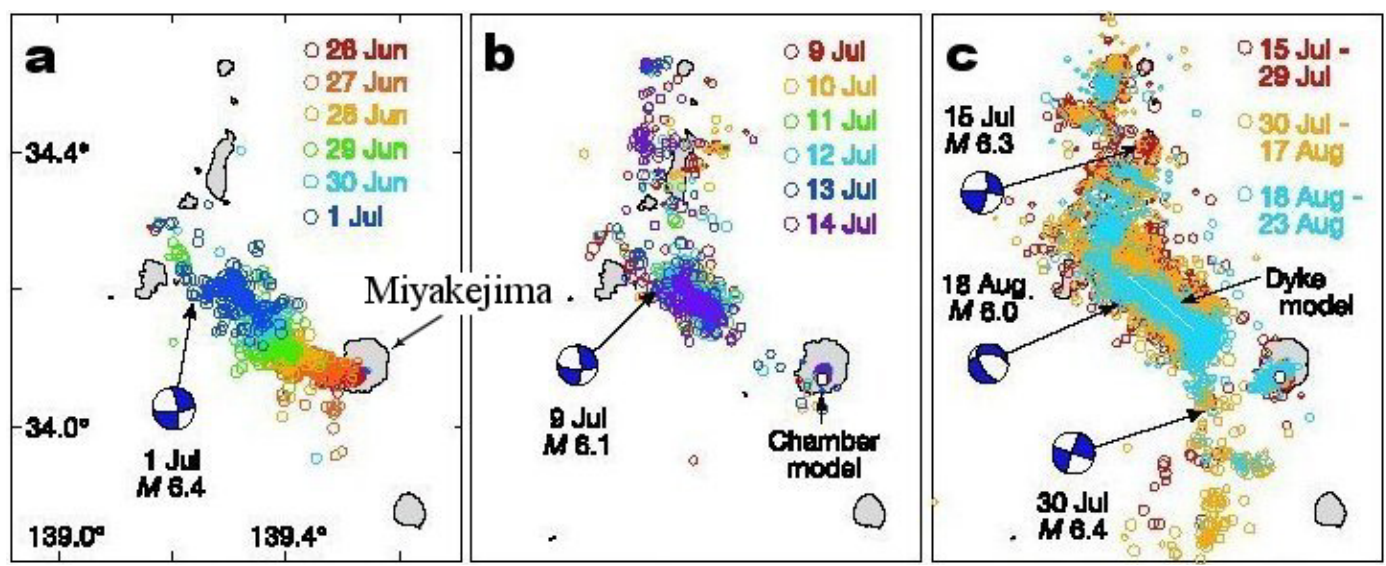

Fig. 1. The dynamics of the Izu earthquakes, 2000. The dates are marked by color. (a), (b) The migration of the EQ epicenters in $N W$ direction provoked by magmatic intrusion of the Oyama volcano. (c) Formation of the large-scale dike (blue color) in $N W$ area of Miyakejima Island.

Table 1. Stations of the magnetic network and magnetic sensors.

\begin{tabular}{ccc}
\hline Station & $\begin{array}{c}\text { IZU NETWORK } \\
\text { Location }\end{array}$ & Magnetic sensor \\
\hline Seikoshi & $34.85^{\circ} \mathrm{N}, 138.82^{\circ} \mathrm{E}$ & MVC-2DS, 50 Hz \\
$\begin{array}{c}\text { Mochikoshi } \\
\text { Kamo }\end{array}$ & $34.89^{\circ} \mathrm{N}, 138.86^{\circ} \mathrm{E}$ & MVC-2DS, 50 Hz \\
& $34.86^{\circ} \mathrm{N}, 138.83^{\circ} \mathrm{E}$ & MVC-1DS, 12.5 Hz \\
& \multicolumn{3}{c}{ BOSO NETWORK } & \\
Station & Location & Magnetic sensor \\
\hline Kiyosumi & $35.16^{\circ} \mathrm{N}, 140.15^{\circ} \mathrm{E}$ & MVC-2DS, 50 Hz \\
Uchiura & $35.16^{\circ} \mathrm{N}, 140.10^{\circ} \mathrm{E}$ & MVC-2DS, 50 Hz \\
Unobe & $35.21^{\circ} \mathrm{N}, 140.20^{\circ} \mathrm{E}$ & MVC-1DS, $12.5 \mathrm{~Hz}$ \\
\hline
\end{tabular}

their migration was considered to be provoked by large-scale dike intrusions of magma.

There were five EQs with magnitude $M>6$ (Fig. 2) detected in the period of the Izu swarm: $M=6.4$ (1 July), $M=6.1$ (9 July), $M=6.3$ (15 July), $M=6.4$ (30 July) and $M=6.0$ (18 August). A complete statistical analysis counted 7000 shocks with magnitude $M \geq 3$ and a total seismic energy release is compatible and even higher than the energy released during the swarm 1965-1967 in Matsushiro, Japan, or in 1980 at Long Value, California. The distance between Kozushima and Shikinejima islands gradually extended by $0.85 \mathrm{~m}$ until 23 August, when the seismicity and rapid displacement ceased in the region (Fig. 1c). Moreover, the swarm was accompanied by five eruptions of Oyama volcano, Miyakejima Island (27 June submarine eruption; a summit eruption on 8 July continued with a large collapse of the crater; phreatic eruptions on 14 and 15 July, large phreatic eruptions on 10 and 18 August; 29 August height of the volcanic smoke elevated up to 8,14 , and $8 \mathrm{~km}$, respectively. A low temperature pyroclastic flow, erupted on August 29 and reached the sea shore). After the eruption, about 4000 peo-

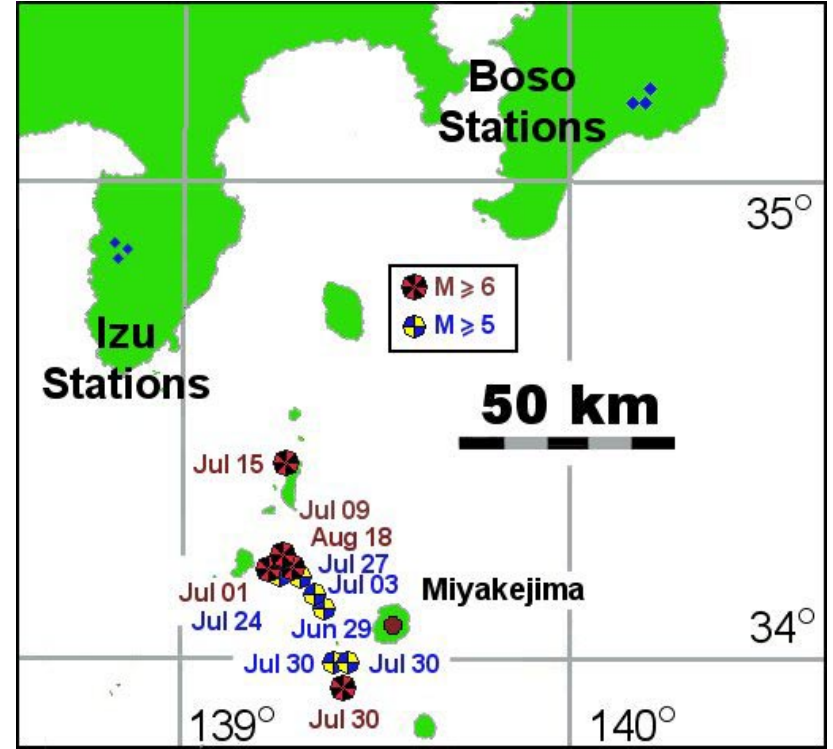

Fig. 2. Station network map and strongest earthquakes. Blue-andyellow circles are moderate EQs with $M_{S}>5$, Red-and-black circles are strong EQs with $M_{S}>6$. The Izu and Boso stations are marked by dark blue rombs.

ple in the island were evacuated and are still living away from the Miyakejima Island. At the present time, $\mathrm{SO}_{2}$ flux still remains at a high level and a weak volcanic glow is observed near the summit crater.

\subsection{Experiment and data processing}

Geomagnetic data were collected since 1 February 2000 until 25 July by 2 networks located at Izu and Boso (Chiba) Peninsulas (Fig. 2) spaced at a distance $140 \mathrm{~km}$ apart. Every network consists of 3 magnetic stations, equipped with 3-component torsion magnetometers MVC-2DS and MVC1DS, operating at $50 \mathrm{~Hz}$ and $12,5 \mathrm{~Hz}$ sampling rates, and a 

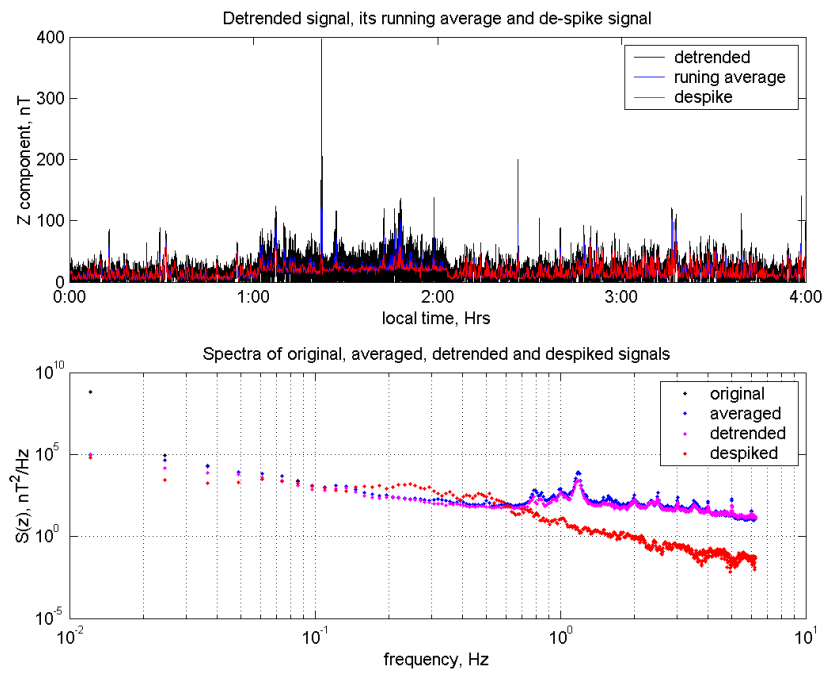

Fig. 3. Demonstration of the de-spike algorithm. Mochikoshi station, 31 December 1999; 4-hours night data. The top pannel shows the already detrended signal (black), its running average (blue) and the de-spiked signal (red). The Bottom pannel shows the original (black), averaged (blue) and detrended (magenta), and de-spiked signals.

GPS system for data synchronization. The network configuration resemble a triangle with distances between stations of $5-8 \mathrm{~km}$. The corresponding coordinates and the type of installed magnetic sensor are shown in Table 1 . The average distance from the EQs swarm hypocenter was about 80$100 \mathrm{~km}$ for the Izu network, and about $130-150 \mathrm{~km}$ for the Boso network.

The new instruments (MVC-2DS) operate at high sampling frequency $(50 \mathrm{~Hz})$. We normally substituted high frequency data with the lower ones $(1 \mathrm{~Hz}$ or $10 \mathrm{~Hz}$ ) utilizing averaging algorithms.

We were seeking to use the whole day record for all the mentioned techniques; the 4-hour data during local nighttime interval (00:00-04:00 a.m.) has been chosen only for the traditional analysis. Such a way normally indicates a risk of noisy content of both the pre-processed signals and the obtained results. Therefore some cleaning operations such as de-spike procedure have been applied to the raw data with the purpose of excluding short-time interference from nearby trains and vehicles. In this procedure we make a running average of the signal, calculate the standard deviation $\sigma$, and normalize the data $B_{i=H, D, Z}$ with the weighting coeeficients $k=\frac{n\langle\sigma\rangle}{(n-1)\langle\sigma\rangle+\sigma_{i}}: B_{i} \rightarrow k B_{i}$ (default value for $n$ is $n=1.3$ ), which is equivalent to cutting high-value spikes of artificial origin (see Fig. 3 for demonstration of the de-spike technique). A possible drawback of this algorythm is that it does not distinguish between the noise and the useful signal and can cut seismogenic EM signal of the pulse form.

The number of points $\left(N_{\mathrm{FFT}}\right)$ for FFT/PSD procedures was 14000 (complete 4-hour interval), for $f=1 \mathrm{~Hz}$ and 140000 , for $f=10 \mathrm{~Hz}$ (time domain $T=4 \mathrm{~h}$, whole day records). The $K p$ index is also plotted (Figs. 4-7 and 9-10 bottom panel).

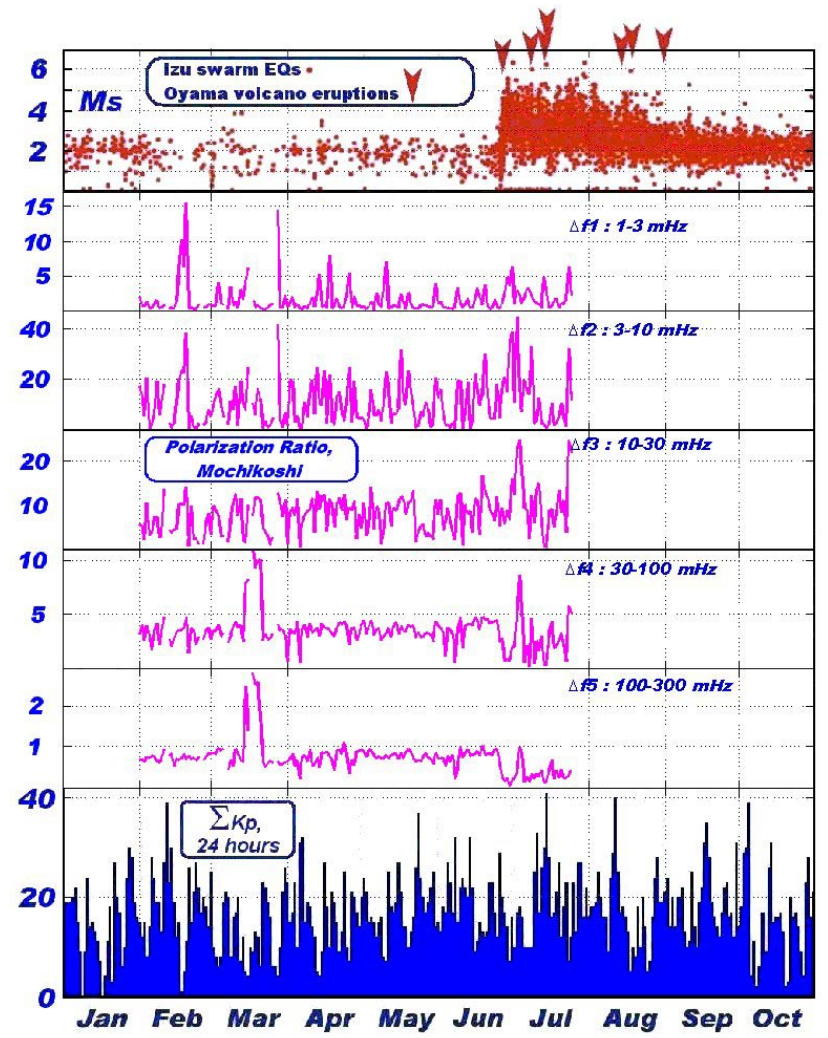

Fig. 4. Polarization ratio variation, Mochikoshi station. The top pannel shows the Oyama volcano eruptions (red arrows); the size of the red circles correspond to the EQ magnitudes. Pannels 2-5 show the polarization ratio dynamics in 5 frequency sub-bands. The bottom pannel shows the sum of the $K p$ index in the corresponding time interval.

The seismic shocks and volcano eruptions at the corresponding time intervals are presented on the upper panel of these figures.

\section{Methodology and analysis of the obtained results}

\subsection{Traditional analysis}

It includes the statistical analysis of the data, obtained from 2 magnetic networks (6 magnetic stations) in 5 frequency subbands with a covered interval $f=1-300 \mathrm{mHz}$.

We calculate spectral densities of 3 magnetic components ( $B_{H}, B_{D}$ and $B_{Z}$ ) in the consecutive $B$ time series, polarization ratios $\left(R=S_{Z} / S_{G}\right.$, where $B_{G}^{2}=B_{H}^{2}+B_{D}^{2}, B_{G}$ being the total horizontal component, $B_{H}$ the horizontal component in the $N S$ direction and $B_{D}$ in the $W E$ direction) and we also construct their derivatives $\left(R\left(\Delta f_{1}\right) / R\left(\Delta f_{2}\right)\right)$.

We combined all the obtained results with the corresponding variation of $\Sigma K p$ calculated for corresponding time intervals with the purpose of isolating the EM emissions generated by seismic sources from global geomagnetic disturbances such as magnetic storms. Figure 4 illustrates the temporal variation of the polarization ratio $R=B_{Z} / B_{H}$ in the 


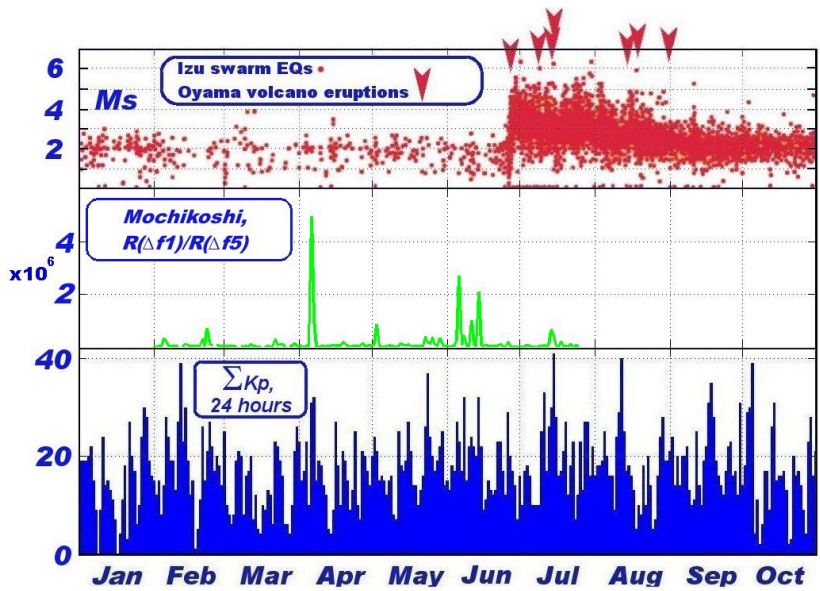

Fig. 5. Spectral ratio $R(\Delta f 1 / \Delta f 5)$ variation, Mochikoshi station. Again at the top the red arrows are Oyama volcano eruptions, and the red circles correspond to the EQ magnitudes. At the middle the spectral ratio $R(\Delta f 1) / R(\Delta f 5)$ dynamics, $\Delta f 1=1-3 \mathrm{mHz}$, $\Delta f 5=100-300 \mathrm{mHz}$ are shown. At the bottom the sum of the $K p$ index in the corresponding time interval is shown.

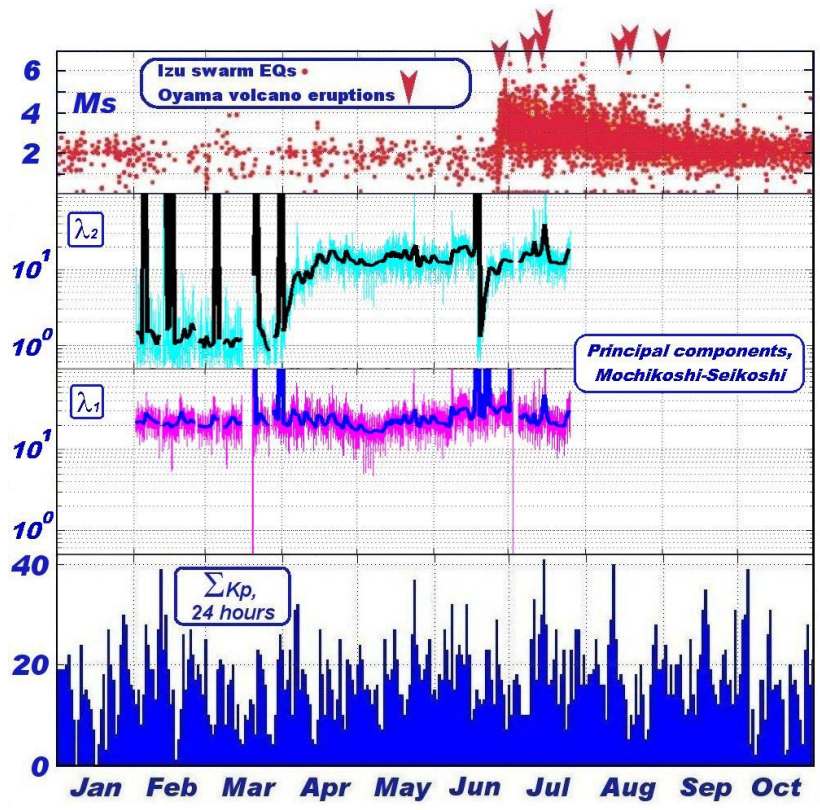

Fig. 6. Principal components $\lambda_{1}$ and $\lambda_{2}$, Izu network, at the frequency interval $\Delta f 2=3-10 \mathrm{mHz}$. Top: Oyama volcano eruptions (red arrows) and EQ magnitudes (red circles). Bottom: the sum of the $K p$ index in the corresponding time interval.

case of Mochikoshi station, which has a low level of industrial noise. The results obtained from the other stations have similar characteristics, although the observed phenomena for the data of Boso stations are relatively weak. Figure 4 reveals a comparatively small rise of the polarization ratio in the frequency bands $\Delta f 2$ and $\Delta f 3$ just before the beginning of the strong seismic and volcanic activity and sharp pre-seismic drop in the bands $\Delta f 4$ and $\Delta f 5$. There were no significant phenomena observed for the frequency inter-

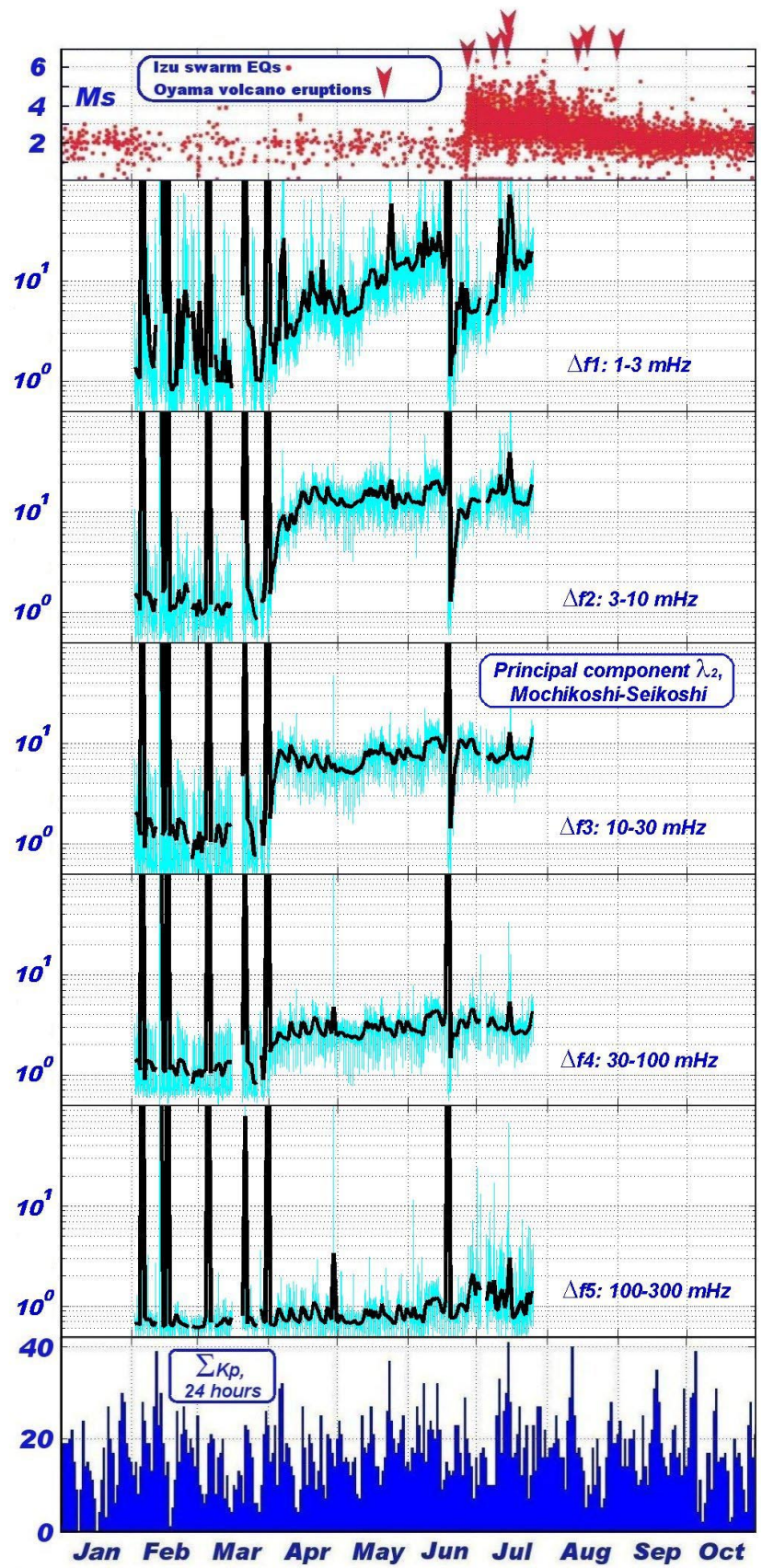

Fig. 7. Dynamics of the principal components $\lambda_{2}$, at 5 frequency sub-bands, Izu stations. Top: Oyama volcano eruptions (red arrows) and EQ magnitudes (red circles). Bottom: the sum of the $K p$ index in the corresponding time interval.

val $\Delta f 1$. Some high spikes at both low and high frequency bands were observed during quiet geomagnetic activity (1819 February, 15-16 and 26-28 March). The spectral ratio $R(\Delta f 1) / R(\Delta f 5)$ expresses the difference in temporal behavior between the polarization ratios at lowest and highest frequencies (Fig. 5) and does not show any obvious relation with seismic activity. On the contrary, its high values appear at periods where seismic activity is weak or absent. 

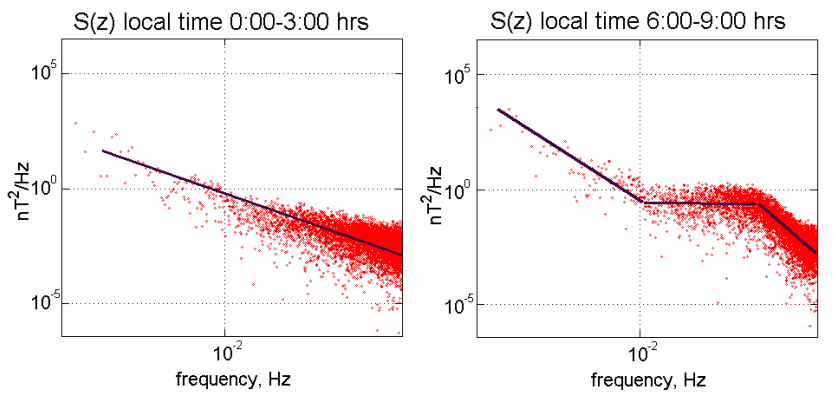

Fig. 8. The mono-fractal ( $1^{\text {st }}$ picture, night interval) and multyfractal $\left(2^{\text {nd }}\right.$ picture, morning) character of the geomagnetic spectra.

\subsection{Principal Component Analysis (PCA)}

The meaning of the PCA analysis is that it can be considered a "black box" which mixes $N$ input signals and resolve them into the same number $N$ of output signals. The characteristic of the input signals is a spacial distibution of observed values (measured in $\mathrm{N}$ closely separated points), and characteristic feature of the output signals is their original statistical properies.

The mathematical formalism of this method is the following. We construct a variance matrix $\mathbf{R}=Y Y^{T}$, where $\mathbf{Y}=\left[y_{1}, y_{2}\right]^{T}, y_{i}=\left[B z_{i}\right](1), B z_{i}(2) \ldots B z_{i}(N), B z i(1) \ldots$ $B z i(N)$ - vertical $(Z)$ component time series for corresponding station $(i)$ for the same $(1 \ldots N)$ time interval. The number of points for this procedure is $N=1800$ (30 min time interval). We used only 2 station data for that convolution set. The principal components are calculated as eigenvalues $\lambda_{1}$, $\lambda_{2}$ from the decomposition of $\mathbf{R}$, where $\mathbf{R}=V \Lambda V^{T}, \Lambda=\left[\lambda_{1}\right.$, $\left.\lambda_{2}\right]$ - eigenvalues matrix, $\mathbf{V}=\left[v_{1}, v_{2}\right]-$ eigenvector matrix for each of the 5 frequency sub-bands mentioned before.

This stage of the PCA is also known as the Blind Deconvolution method (Hattori et al., 2004a). The best application of this method for the geomagnetic data ( $H$-component of magnetic field, 3 station data set) has been made by Hattori et al. (2004b) for the 3 station data set. The obtained results were classificated as of space origin $\left(\lambda_{1}\right)$, noise component $\left(\lambda_{2}\right)$ and finally, the seismogenic signal $\left(\lambda_{3}\right)$.

As we have chosen only 2 components for our analysis, the statistical resolution of the obtained results was not so successful because both output signals have been noise contaminated. The temporal evolution of the obtained 2 principal components $\lambda_{1}$ and $\lambda_{2}$ for the Izu network in a frequency interval $\Delta f 2(3-10 \mathrm{mHz})$ is illustrated by Fig. 6 . The thick line relates to values averaged over a 1 day interval (48 points). The dynamics of the principal component $\lambda_{2}$, classified as seismic related, is shown in Fig. 7 for every frequency interval where it was calculated. A common feature of the low frequency curves is a peak immediately followed by an abrupt drop. Another tendency has been found in the temporal behavior at the lowest frequency $\Delta f 1$ : the component grows exponentially (log scale) from the middle of March to 19 June, sharply rises and drops at 19 June and then continue

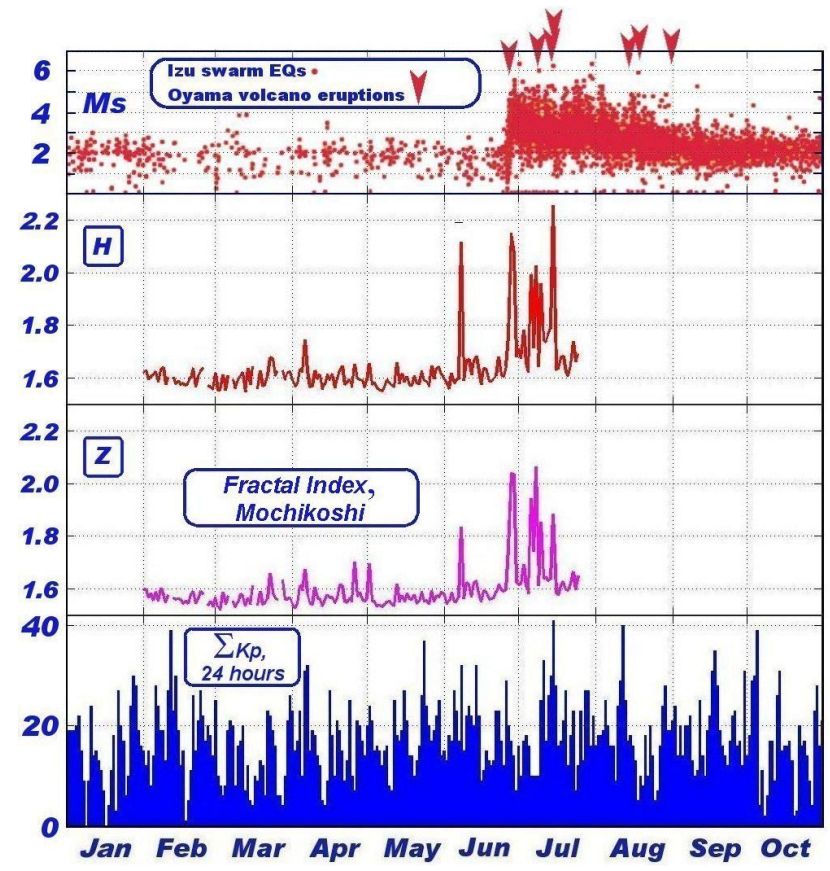

Fig. 9. Fractal index $\beta$ variation, Mochikoshi station. $H$ and $Z$ components. Top: Oyama volcano eruptions (red arrows) and EQ magnitudes (red circles). Bottom: the sum of the $K p$ index in the corresponding time interval.

its exponential growth with a higher rate. The presence of 5 high-level spikes in the interval February-March still remains unexplained. It should be noticed that the PCA results obtained from Boso station expressed much weaker tendencies and the exponential raise in the lowest frequency band was not at all observed, in contrast to the Izu stations results.

\subsection{The fractal analysis}

This analysis is based on the conception of Self-Organized Criticality (SOC) which describes the autoaffine processes. In our case, the spectrum of the geomagnetic signal satisfies the power law $S \sim f^{-\beta}$. According to the phenomenological model (Troyan et al., 1999) by means of changes of the exponent index $\beta$ (fractal index) we can evaluate the state of the system (from the chaotic to the critical) which can change significantly just before the earthquake. This has been shown for geomagnetic series (Smirnova et al., 2004) as well as for electrical series (Ramirez-Rojas et al., 2004). The $\beta$ itself also desribes the statistical character of different processes such like white noise $(\beta=0)$, Flicker Noise $(\beta=1)$ and Brownian Motion $(\beta=2)$.

The calculation of the fractal index $\beta$ can be made in different ways. The evaluation of the methods have been done by Ghoto et al. (2004). We have chosen the FFT method. As the law is $S \sim f^{-\beta}$, this procedure requires the graph of daily $S_{H, D, Z}=S_{H, D, Z}(f)$ to be presented in a logarithmic scale for both X- and Y-axes. We calculate the exponent $\beta$ by linear fit of the plotted dependence, using the least-squares 


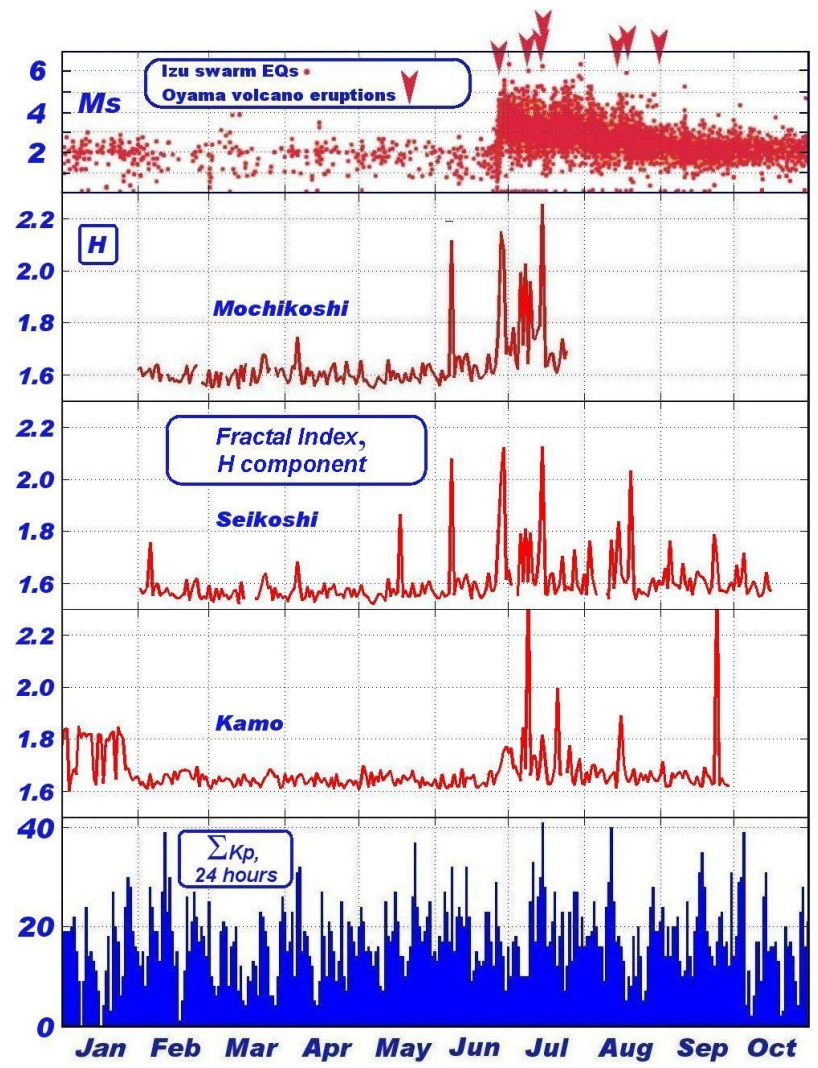

Fig. 10. Fractal index $\beta$ variation for 3 Izu network stations, $H$ component. Top: Oyama volcano eruptions (red arrows) and EQ magnitudes (red circles). Bottom: the sum of the $K p$ index in the corresponding time interval.

method. Usually the shape of the the spectra is quite different for the data obtained from different regions. Sometimes it has fractures and bends at definite frequencies, both for certain periods of time and for the whole day record (see Fig. 8) which can be characterized as a multy-fractal property of the spectra. In the case of IZU data, the shape of the spectra is quite smooth and does not contain significant bends (mostly monofractal dependence) and therefore we use the whole frequency interval for the linear fit interpolation.

The temporal variation of the fractal index for the vertical and horizontal components, calculated by FFT method, is presented for the Mochikoshi station data in Fig. 9. The presented temporal variation of the $\beta$ dependences have similar behavior especially at the time intervals preceding and during the seismic and volcanic activities. We selected the $H$-component to compare the results obtained from different stations. It can be clearly seen from Fig. 10 that all the curves reveal a significant growth of $\beta$ at the times before and during the earthquakes although the pre-seismic growth of the fractal index, calculated for the Kamo station, is much weaker and smoother. This can possibly be explained as a technical effect of the down-sampling procedures from $50 \mathrm{~Hz}$ to $10 \mathrm{~Hz}$, forthe Mochikoshy and Seikoshi stations data, which normally perturbs such a characteristics as the "rugosity" of the spectra and affects the $\beta$ accordingly.

Also, it is difficult to explain the relatively high level of $\beta$ in January 2000. Unfortunately we cannot check the behavior of fractal index by calculating it for other stations data, because of a lack of data.

\section{Conclusions}

The investigation of the ULF electromagnetic activity preceeding the IZU EQ swarm and Oyama volcano eruption in the summer of 2000 reveals certain peculiarities: Noticeable drops of polarization at high frequency bands (30-100 and $100-300 \mathrm{mHz}$ ) just before the swarm begins, and a slight increase at lower frequencies (3-10 and $10-30 \mathrm{mHz}$ ) approximately one month before the event; an exponential growth of the lowest frequency part of the principal component up to 1 week before the seismic activity with a peak and sharp drop, simultaneously occurring at other frequency bands;

perceptible spikes 2 weeks before and just before the events in the temporal variation of fractal index, continued by noticeable growth at the time of high seismic activity, which could be interpreted as possible precursors of earthquakes.

The man-made noise contamination is much higher then the natural signal, and so is the electromagnetic emission associated with seismic events. Therefore, additional processing for ULF emission separation is required.

Acknowledgements. The authors are grateful to $\mathrm{S}$. Toda for seismological data, pictures and discussions and to K. Hattori and to another anonymous referee for constructive criticisms and comments that have given us the possibility to improve the text of this manuscript.

Edited by: P. F. Biagi

Reviewed by: K. Hattori and another referee

\section{References}

Fraser-Smith, A. C., Bernardy, A., McGill, P. R. et al.: Low frequency magnetic field measurements near the epicenter of the Loma Prieta earthquake, Geophys. Res. Lett., 17, 1465-1468, 1990.

Gotoh, K., Hayakawa, M., Smirnova, N. and Hattori, K.: Fractal analysis of seismogenic ULF emissions, Phys. Chem. Earth, 29, 419-424, 2004.

Hattori, K., Takahashi, I., Yoshino, C. et al.: ULF geomagnetic field measurements in Japan and some recent results associated with Iwateken Nairiku Hokubu Earthquake in 1998, Phys. Chem. Earth, 29, 481-494, 2004a.

Hattori, K., Serita, A., Gotoh, K. et al.: ULF geomagnetic anomaly associated with 2000 Izu islands earthquake swarm, Japan, Phys. Chem. Earth, 29, 425-436, 2004 b.

Hayakawa, M., Molchanov, O. A., Ondoh, T., and Kawai, E.: Precursory signatures of the Kobe Earthquake on VLF Subionospheric signals, J. Atmos. Electr., 16, 3, 247-257, 1996.

Ismaguilov, V., Kopytenko, Yu., Hattori, K., et al.: ULF magnetic emissions connected with under sea bottom earthquakes, Nat. Haz. Earth Syst. Sci., 1, 23-31, 2001,

SRef-ID: 1684-9981/nhess/2001-1-23. 
Ramirez-Rojas, A., Pavia-Miller, C. G., and Angulo-Brown, F.: Statistical behavior of the spectral exponent and the correlation time of electric self-potential time series associated to the $M s=7.4,14$ September 1995 earthquake in Mexico, Phys. Chem. Earth, 29, 4-9, 305-312, 2004.

Smirnova, N., Hayakawa, M., Gotoh, K., and Volobuev, D.: Scaling characteristics of ULF geomagnetic fields at the Guam seismoactive area and their dynamics in relation to the earthquake, Nat. Haz. Earth Syst. Sci., 1, 119-126, 2001,

\section{SRef-ID: 1684-9981/nhess/2001-1-119.}

Smirnova, N., Hayakawa, M., and Gotoh, K.: Precursory behavior of fractal characteristics of the ULF electromagnetic fields in seismic active zones before strong earthquakes, Phys. Chem. Earth, 29, 445-451, 2004.

Troyan, V. N., Smirnova, N. A., Kopytenko, Yu. A., Peterson, Th., and Hayakawa, M.: Development of a complex approach for searching and investigation of electromagnetic precursors of earthquakes: organization of experiments and analysis procedures, in Atmospheric and Ionospheric electromagnetic phenomena associated with Earthquakes, edited by Hayakawa, M., Terra Scientific Publishing Company, Tokyo, 147-171, 1999.
Toda, S., Stein, R. S., and Sagiya, T.: Evidence from the AD 2000 Izu islands earthquake swarm that stressing rate governs seismicity, Nature, 419, 58-61, 2002.

Uyeda, S., Nagao, T., Orihara, Y. et al.: Geoelectrical potential changes: possible precursors to earthquakes in Japan, Proc. Nat. Acad. Sci., 97, 4561-4566, 2000.

Varotsos, P., Lazaridou, M., Eftaxias, K. et al.: Short term earthquake prediction in Greece by seismic electric signals, Critical review on VAN, edited by Lighthill, J., Word Scientific, Singapore, 29-76, 1996. 